\title{
Improving the Interoperation between Generics Translations
}

\author{
Vlad Ureche Milos Stojanovic Romain Beguet Nicolas Stucki Martin Odersky \\ École polytechnique fédérale de Lausanne, Switzerland \\ $\{$ first.last\}@epfl.ch
}

\begin{abstract}
Generics on the Java platform are compiled using the erasure transformation, which only supports by-reference values. This causes slowdowns when generics operate on primitive types, such as integers, as they have to be transformed into reference-based objects.

Project Valhalla is an effort to remedy this problem by specializing classes at load-time so they can efficiently handle primitive values. In its current early prototype ${ }^{1}$, the Valhalla compilation scheme limits the interaction between specialized and erased generics, thus preventing certain useful code patterns from being expressed.

Scala has been using compile-time specialization for 6 years and has three generics compilation schemes working side by side. In Scala, programmers are allowed to write code that freely exercises the interaction between the different compilation schemes, at the expense of introducing subtle performance issues. Similar performance issues can affect Valhalla-enabled bytecode, whether the code was written in Java or translated from other JVM languages.

In this context we explain how we help programmers avoid these performance regressions in the miniboxing transformation: (1) by issuing actionable performance advisories that steer programmers away from performance regressions and (2) by providing alternatives to the standard library constructs that use the miniboxing encoding, thus avoiding the conversion overhead.
\end{abstract}

Keywords generics, specialization, miniboxing, backward compatibility, data representation, performance, Java, bytecode, JVM

\section{Introduction}

Generics on the Java platform are compiled using the erasure transformation [12], which allows them to be fully backward compatible with pre-generics bytecode. Unfortunately, this also means that they only handle by-reference values (objects) and not primitive types. Thus, primitive values such as bytes and integers have to be converted to heap objects each time they interact with generics. This conversion, known as boxing, compromises the execution performance and increases the heap footprint, forcing Java to lag behind lower-level languages such as $\mathrm{C}$ or $\mathrm{C}++$.

\footnotetext{
${ }^{1}$ As of August 2015 [19, 20].
}

Permission to make digital or hard copies of all or part of this work for personal or classroom use is granted without fee provided that copies are not made or distributed for profit or commercial advantage and that copies bear this notice and the full citation on the first page. Copyrights for components of this work owned by others than the author(s) must be honored. Abstracting with credit is permitted. To copy otherwise, or republish, to post on servers or to redistribute to lists, requires prior specific permission and/or a fee. Request permissions from permissions@acm.org.

PPPJ '15, September 08 - 11, 2015, Melbourne, FL, USA.

Copyright is held by the owner/author(s). Publication rights licensed to ACM.

ACM 978-1-4503-3712-0/15/09...\$15.00.

http://dx.doi.org/10.1145/2807426.2807436
The performance drawbacks of erasure are currently being addressed in Project Valhalla ${ }^{2}$, an important undertaking led by the Java platform architects and aimed at providing unboxed generics for Java and other JVM languages. The updated bytecode format in Project Valhalla will include the necessary type information to allow load-time class specialization, effectively creating different versions of classes that directly support primitive types. This loadtime transformation approach is also employed by the .NET framework $[21,36]$ in order to implement generics.

Unlike .NET generics, which are always specialized, the current design of Project Valhalla, as of August 2015, makes it an explicit goal to have specialization as an opt-in transformation. This will allow the ecosystem to evolve smoothly from erased to specialized generics, allowing both erased and specialized classes to work side by side. However, in the current early prototype, there are still some restrictions: (1) erased code cannot handle specialized instances in a generic manner and, (2) abstracting over specialized classes using wildcard types $[19,20]$ pays the cost of boxing primitive types. This is shown in the following example:

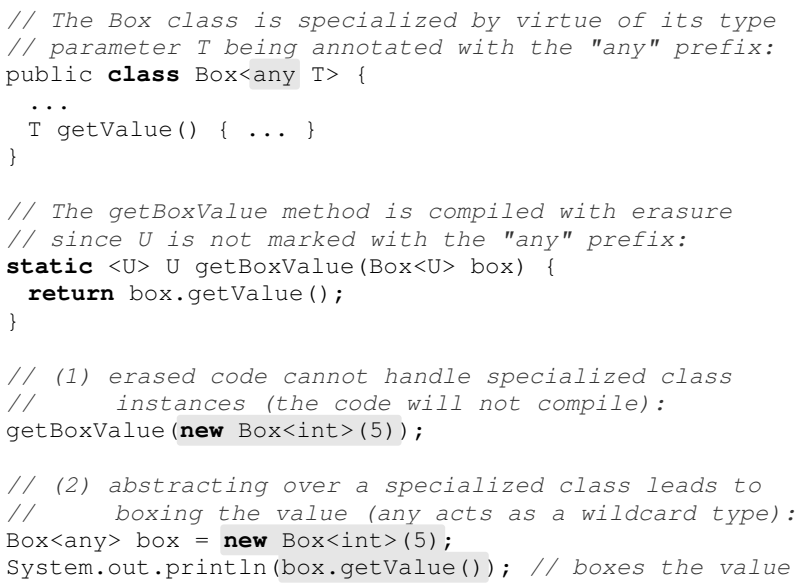

These two code patterns could easily be rewritten to make the code compile and to avoid the overhead of boxing. For the first pattern, adding the any prefix to the type parameter $\mathrm{U}$ of method getBoxValue would make it specialized as well, allowing it to handle the incoming argument. In the second pattern, the wildcard, which is equivalent to extending Box<?> to value types, could be replaced by the exact type, Box<int $>$, eliminating the overhead of boxing. Yet, in the general case, not all code can be changed at will, due to interactions, backward compatibility or because it resides in external libraries. Thus, a better solution would be to have erased and specialized generics interoperate, ideally without the overhead.

\footnotetext{
2 The Valhalla Project is still in its infancy, but early prototypes are openly available and the hard goals have been clearly defined in $[18,25,26]$.
} 
The Scala programming language, which also compiles to JVM bytecode, has had compile-time specialization for 6 years [13, 14] and currently has three mechanisms for compiling generics: erasure, specialization and a new arrival, miniboxing [31]. In Scala, all three generics compilation schemes can be freely mixed:

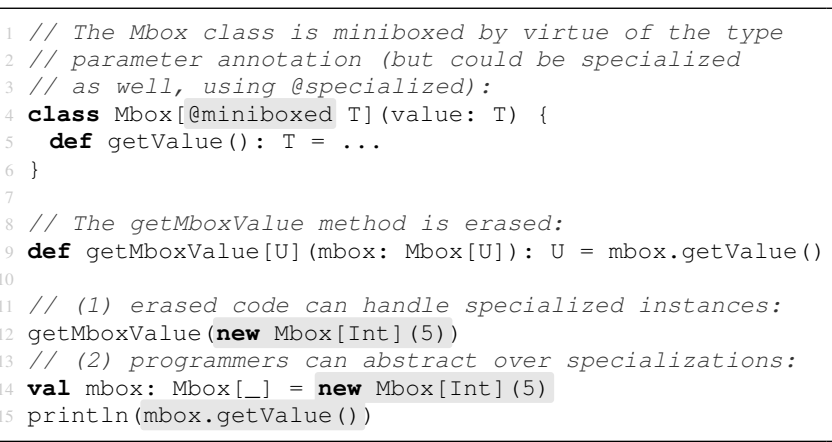

Despite the uniform behavior, Scala does pay a hefty price for being able to freely mix code using the three generics compilation schemes: calls between different compilation schemes require boxing primitive values. The reason is that only boxed primitive values are understood by all three transformations. Furthermore, as we will see later on, instantiating a miniboxed (or specialized) class from erased code leads to the erased version being instantiated instead of its miniboxed (or specialized) equivalent, in turn leading to unexpected performance regressions.

In this paper, we show how we completely eliminate the unexpected slowdowns in the miniboxing transformation and, as a side effect, allow programmers to easily and robustly use miniboxing to speed up their programs. The underlying property we are after is that, inside hot loops and performance-sensitive parts of the program, all generic code uses the same compilation scheme, in this case, miniboxing. This way, primitive types are always passed using the same data representation, whether that's the miniboxed encoding (for miniboxing) or the unboxed representation (for specialization).

We show two approaches for harmonizing the compilation scheme across performance-sensitive code:

Issuing actionable performance advisories when compilation schemes do not match, allowing the programmer to harmonize them. For example, when a generic method takes a miniboxed class as a parameter and tries to call methods on it, we automatically generate performance advisories:

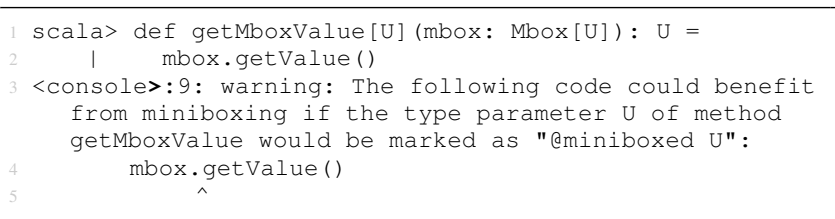

Another problem that occurs frequently concerns library evolution: as a new compilation scheme arrives, it is best if all libraries start using it as soon as possible. However, backward compatibility prohibits changing the compilation scheme for the standard library, as it would break old bytecode. In Scala, we had this problem because many of the core language constructs, such as functions and tuples use specialization instead of miniboxing. Similarly, Java has as many as 20 manual specializations for the arity 1 lambda, such as IntConsumer, IntPredicate and so on. Replacing these by a single specialized functional interface would be desirable, but is realistically impossible. We present a solution for this:
Efficiently bridging the gap between compilation schemes. In the case of miniboxing, which is a compiler plugin, we were not able to change the Scala standard library functions and tuples to use the miniboxing scheme. Instead, we describe the approaches we use to efficiently communicate to the existing library classes, and, where necessary, to replace them by miniboxed equivalents.

With this, the paper makes four key contributions to the Java community and, in the general sense, to the field of compiling objectoriented languages with generics:

- Describing the problems involved in mixing different generics compilation schemes $(\$ 2)$;

- Describing a general mechanism for harmonizing the compilation scheme (\$3);

- Describing the approaches we use to fast-path communication between different generic compilation schemes $(\$ 4)$;

- Validating the approach using the miniboxing plugin (\$5).

The evaluation section ( $\$ 5$ ) shows that warnings not only help avoid performance regressions, but can also guide developers into further improving their program's performance.

\section{Compilation Schemes for Generics}

This section describes the different compilation schemes for generics in Scala. We mainly use Scala for the examples, but the discussion can be applied to Java as well. Differences between Scala specialization and Project Valhalla are pointed out along the way.

\subsection{Erasure in Scala}

The current compilation scheme for generics in both Java and Scala is called erasure, and is the simplest compilation scheme possible for generics. Erasure requires all data, regardless of its type, to be passed in by reference, pointing to heap objects. Let us take a simple example, a generic identity method written in Scala:

def identity[T] $(t: T): T=t$

val five $=$ identity $(5)$

When compiled, the bytecode for the method is ${ }^{3}$ :

def identity (t: Object): Object $=t$

As the name suggests, the type parameter T was "erased" from the method, leaving it to accept and return object, T's upper bound. The problem with this approach is that values of primitive types, such as integers, need to be transformed into heap objects when passed to generic code, so they are compatible with object.This process, called boxing goes two ways: the argument of method identity needs to be boxed while the return value needs to be unboxed back to a primitive type:

val five = identity (Integer.valueof(5)). intValue()

Boxing primitive types requires heap allocation and garbage collection, both of which degrade program performance. Furthermore, when values are stored in generic classes, such as vector $[\mathrm{T}]$, they need to be stored in the boxed format, thus inflating the heap memory requirements and slowing down execution. In practice, generic methods can be as much as 10 times slower than their monomorphic (primitive) instantiations. This gave rise to a simple and effective idea: specialization.

\footnotetext{
${ }^{3}$ Throughout the paper, we show the source-equivalent of bytecode. The context clarifies whether we are showing source code or compiled bytecode
} 


\subsection{Specialization}

Specialization $[13,14]$ is the second approach used by the Scala compiler to translate generics and, for methods, is similar to Project Valhalla. It is triggered by the @specialized annotation:

def identity[@specialized $T$ ] ( $t: T): T=t$

val five = identity (5)

Based on the annotation, the specialization transformation creates several versions of the identity method:

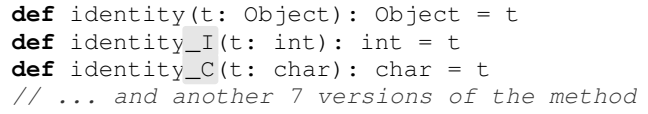

Having multiple methods, also called specialized variants or simply specializations of the ident ity method, the compiler can optimize the call to identity:

val five: int $=$ identity_I (5)

This transformation side-steps the need for a heap object allocation, improving the program performance. However, specialization is not without limitations. As we have seen, it creates 10 versions of the method for each type parameter: the reference-based version plus 9 specializations (Scala has the 8 primitive types in Java plus the Unit primitive type, which corresponds to Java's void). And it gets worse: in general, for $N$ specialized type parameters, it creates $10^{N}$ specialized variants, the Cartesian product covering all combinations.

Lacking Project Valhalla's virtual machine support, Scala specialization generates the specialized variants during compilation and stores them as bytecode. This prevents the Scala library from using specialization extensively, since many important classes have one, two or even three type parameters. This led to the next development, the miniboxing transformation.

\subsection{Miniboxing}

Taking a low level perspective, we can observe the fact that all primitive types in the Scala programming language fit within 64 bits. This is the main idea that motivated the miniboxing transformation [31]: instead of creating separate versions of the code for each primitive type alone, we can create a single one, which stores 64-bit encoded values, much like C's untagged union. The previous example:

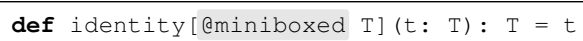

Is compiled ${ }^{4}$ to the following bytecode:

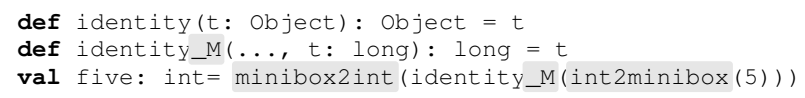

Alert readers will notice the minibox 2 int and int 2 minibox transformations act exactly like the boxing coercions in the case of erased generics. This is true: the values are being coerced to the miniboxed representation, much like boxing in the case of erasure. Yet, our benchmarks on the Java Virtual Machine platform have

\footnotetext{
${ }^{4}$ In the rest of the paper we assume the miniboxing Scala complier plugin is active unless otherwise noted. For more information on adding the miniboxing plugin to the build please see http://scala-miniboxing.org.
}

shown that the miniboxing conversion cost is completely eliminated when just-in-time compiling to native 64-bit code. Further benchmarking has shown that the code matches the performance of specialized code within a $10 \%$ slowdown due to coercions [31], compared to a 10x slowdown in the case of boxing.

There is an ellipsis in the definition of the identity_M method, which stands for what we call a type byte: a byte describing the type encoded in the long integer, allowing operations such as tostring, hashcode or equals to be executed correctly on encoded values:

def string[@miniboxed $\mathrm{T}](t: \mathrm{T})$ : String = t.toString

In order to transform this method, we need to treat the primitive value as its original type (corresponding to $\mathrm{T}$ ) rather than a long integer. To do so, we use the type byte:

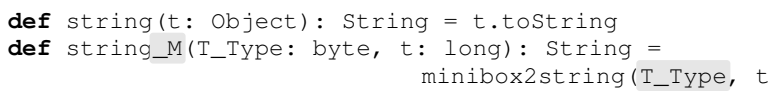

Then, when the programmer makes a call to string:

string[Boolean] (true)

It automatically gets transformed in the compiler pipeline to:

string_M(BOOL, bool2minibox(true))

Knowing the type byte, the minibox2string can do its magic: decoding the long integer into a "true" or "false" string, depending on the encoded value. Although seemingly simple, the code transformation to implement the miniboxing transformation is actually rather tricky [17, 32, 33].

So far, we have only looked at methods, but transforming classes poses even greater challenges.

\subsection{Class Transformation in Project Valhalla}

Project Valhalla takes a straight-forward approach to specialization: classes are duplicated and all previous references to the type parameters are transformed. Given the linked list node class:

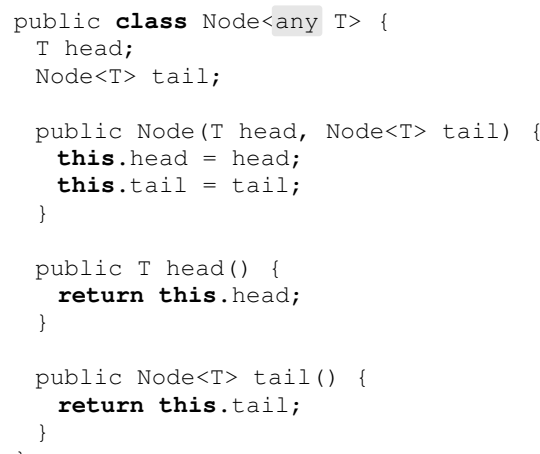

When specializing the Node class for the int primitive type, Project Valhalla employs its custom classloader to clone and adapt the bytecode for class Node. Among other transformations, it replaces references to $\mathrm{T}$ by int [18]:

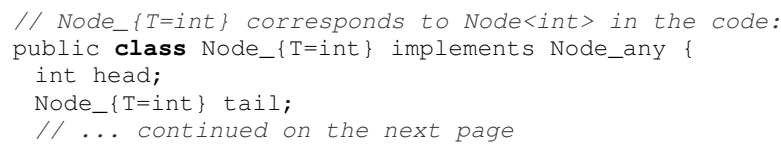




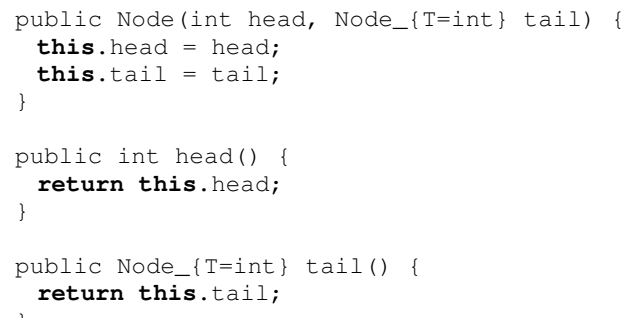

The load-time translation produces Node_ $\{\mathrm{T}=$ int $\}$, which handles unboxed int values. The fact that Node_ $\{\mathrm{T}=i n t\}$ is not a subclass of Node negatively impacts the interoperation with erasure. Let us take an example method, compiled with erasure:

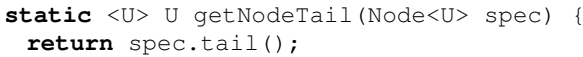

The corresponding bytecode is:

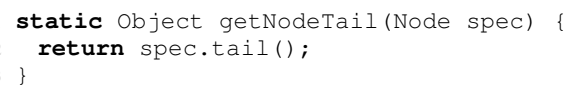

Since Node_\{T=int $\}$ is not a subclass of Node, the getNodeTail method cannot handle specialized instantiations of Node as arguments, forcing the compiler to reject code patterns such as getNodeTail (new Node<int> (...)). This limits the interaction between erased and specialized generics and prevents many useful code patterns from being expressed. In turn, it prolongs the time necessary to adopt specialization: if a single generic library is compiled with erasure, its clients need to use erasure as well, otherwise they would not be able to use that library.

Acknowledging the importance of allowing erased methods to handle specialized instances, a solution was later introduced: In the initial prototype, as of December 2014, the only common parent of classes Node and Node_\{ $\mathrm{T}=$ int $\}$ was object. However, as of August 2015, the translation has been improved to automatically introduce the Node_any interface which serves as a common supertype of Node and its specializations (e.g. Node_\{T=int $\}$ ). Aside from simplifying the translation from JVM languages to Valhalla bytecode, this change also enables programmers to abstract over specialized classes:

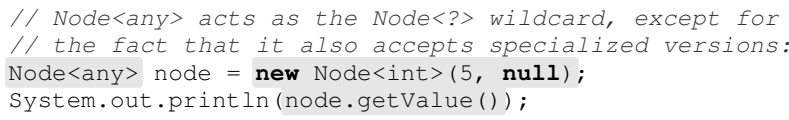

This example is translated to the following bytecode:

Node_any node $=$ new Node_ $\{\mathrm{T}=$ int $\}(5$, null $)$;

System.out.println (node.getvalue()/* of type object */)

The Node_any interface is called an "erased view", since it enables accessing the specialized class in a uniform, erased-like manner. Since there is no mechanism to return either a reference or a primitive type (corresponding to the any wildcard), the call to getValue () boxes the value returned. Thus, the call introduces a silent performance regression, which is the cost of interoperability.

The miniboxing translation allows erased, specialized and miniboxed code to freely interact, enabling both code patterns above, at the expense of adding even more boxing operations.

\subsection{Class Transformation in Miniboxing}

Scala specialization $[13,14]$ introduced a better class translation, which is compatible to erased generics. Miniboxing [31] inherited and adapted this scheme, addressing two of its major drawbacks, namely the double fields and broken inheritance. For this reason, we will present the miniboxed class translation scheme directly.

The main challenge of interoperating with erased generics is to preserve the inheritance relation while providing specialized variants of the class, where fields are encoded as miniboxed long integers instead of ob jects. Let us take the linked list node class again, this time written in Scala:

class Node[@miniboxed T] (val head:T, val tail:Node[T])

The Scala compiler desugars the class to (some aspects omitted):

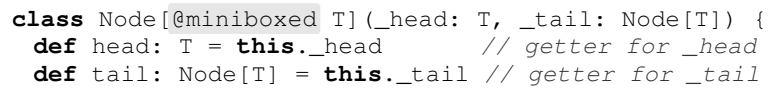

There are three subtleties in the Node translation:

- First, there should be two versions of the class: one where _head is miniboxed, called Node_M and another one where _head is an object, called Node_L;

- Then, types like Node [_], which corresponds to Java's wildcard Node $<$ any $>$ can be instantiated by both classes, so the two need to share a common interface, the "erased view";

- Finally, this shared interface has to contain the specialized accessors corresponding to both classes (so both classes should implement all the methods).

Given these constraints, miniboxing compiles Node to an interface:

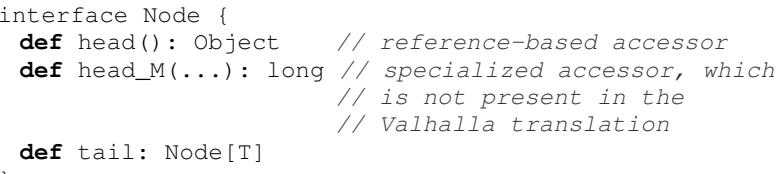

Note that the tail method does not have a second version, as it doesn't accept or return primitive values. Then, we have the two specialized variants of class Node:

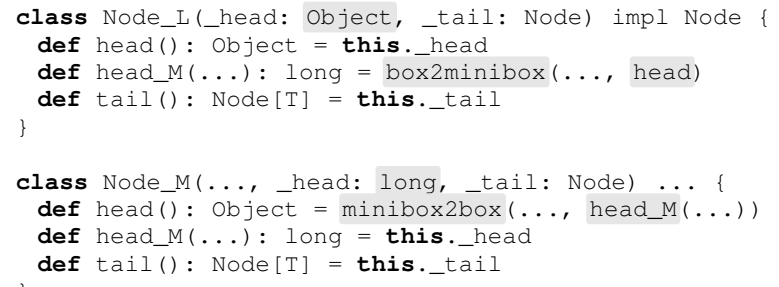

As before, the ellipsis corresponds to the type bytes. With this translation, code that instantiates the Node class is automatically transformed to use one of the two variants. For example:

new Node [Int] $(4$, null $)$

Is automatically transformed to:

new Node_M[Int] (INT, int2minibox(4), null)

And, when Node is instantiated with a miniboxed type parameter: 
def newNode[ [miniboxed $\mathrm{T}](\mathrm{t}: \mathrm{T})=$

new Node $[T]$ ( $t$, null)

The code is translated to:

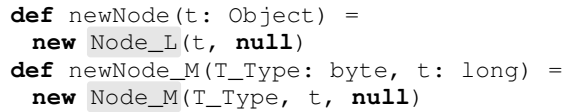

The translation hints at an optimization that can be done: given a value of type Node $[\mathrm{T}$ ] where $\mathrm{T}$ is either a primitive or known to be miniboxed, the compiler can call head_M instead of head, skipping a conversion. The following code:

val $\mathrm{n}=$ new Node [Int] $(3$, null $)$

n.head

Is translated to:

val $\mathrm{n}=$ new Node $\mathrm{M}(\ldots, 3$, null $)$

$\mathrm{n}$.head_M (...)

The rewrite also occurs when the type argument is miniboxed:

def getFirst[@miniboxed $\mathrm{T}](\mathrm{n}$ : Node $[\mathrm{T}])=\mathrm{n} \cdot$ head

This method is translated to:

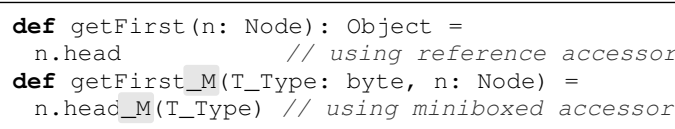

At this point, you may be wondering why the getFirst bytecode receives a parameter of type Node instead of Node_L, or, respectively, Node_M. The reason is interoperability with erased generics.

\subsection{Interoperating with Erased Generics}

So far, we have seen the following two invariants:

- we call the head_M accessor on receivers of type Node[T] where $\mathrm{T}$ is either miniboxed or is a primitive type, optimistically assuming the receiver is an instance of class Node_M;

- otherwise, we call the head accessor, assuming the receiver is an instance of class Node_L.

Unfortunately, interoperating with erased generics violates both invariants. Consider the following method:

def newNodeErased $[\mathrm{T}]$ (head: $\mathrm{T}$ ) =

new Node $[\mathrm{T}]$ (head, null)

During the compilation of this method, using to erased generics, the compiler is forced to make a static (compile-time) choice: Which class to instantiate for the new Node $[\mathrm{T}]$ ?

Since newNodeErased can be called with both (boxed) primitives and objects, the only valid choice is Node_L, which can handle both cases. Contrarily, Node_M can't handle references, since object pointers are not directly accessible in the JVM. Thus, we have:

def newNodeErased(head: Object) =

new Node_L(head, null)

Which allows the erased generics to invalidate the invariants:

val $\mathrm{n}:$ Node [Int] = newNodeErased [Int] (3)

$\mathrm{n}$.head // $\mathrm{n}:$ Node $[$ Int] $=>$ call head_M val $\mathrm{n}:$ Node $=$ newNodeErased $(\ldots)$ // retuns a Node I n.head_M(INT) // assumption: receiver has type Node_M

This way, the call to head_M occurs on a Node_L instance. The symmetric case can also occur, calling head on a Node_M class. And, what is worse, we can end up with a Node_L class storing a primitive value, which means it will be boxed.

Fortunately, by never promising more than the erased view, Node, the compilation scheme is robust enough to handle the mix-up. This allows correctly compiling both patterns in the Valhalla example:

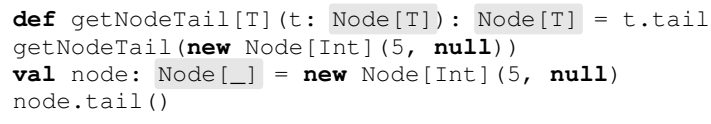

By producing the following bytecode:

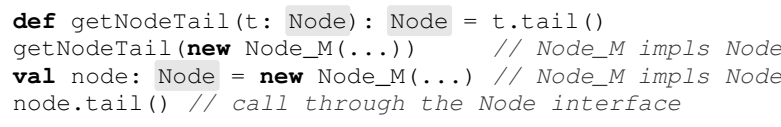

Comparing to Valhalla bytecode, the Node interface corresponds to Valhalla's Node_any interface, the "erased view". Yet, by replacing references to Node $[\mathrm{T}]$ by the erased view (instead of the Node_I class) miniboxing allows better interoperation.

\section{Performance Advisories}

The previous section has shown that, when used globally, miniboxed generics provide two key invariants that ensure primitive values are always passed using the miniboxed (long integer) encoding:

- Instantiations of miniboxed classes use the most specific variant (e.g. a value of type Node [ Int ] has runtime class Node_M);

- Methods called on a miniboxed class use the most specific specialization available (e.g. a runtime class Node_M always receives calls to the miniboxed head_M accessor)

The presence of erasure and wildcard-type abstractions (such as Node [_] ) leads to violations of these two invariants: the reference variant of a miniboxed class may be instantiated in place of a miniboxed variant or the method called may not be the most specific one available. In both cases, the compilation scheme is resilient, producing correct results, at the expense of performance regressions, caused by boxing primitive types.

There key to avoiding these subtle performance regressions is to intercept the class instantiations and method calls that violate the invariant and report actionable advisories to the users, in the form of compiler warnings. Luckily, all the information necessary to detect invariant violations is available during compilation.

\subsection{Performance Advisories Overview}

Advisories are most commonly triggered by interacting with erased or specialized generics, but can also be caused by technical or design limitations. There are as many as ten different performance advisories implemented in the miniboxing plugin, but in order to focus on the concept, we will only look at the three most common advisories, two of which are caused by the interaction with erased generics. To show exactly how the slowdowns occur, we can take the following piece of code:

def foo[Aminiboxed T] ( $t: T): T=\operatorname{bar}(t)$

def bar[@miniboxed U] ( $U$ : $U): U=b a z(u)$

def baz[@miniboxed V] (v: V): $\mathrm{V}=\mathrm{V}$ 
The code is compiled to:

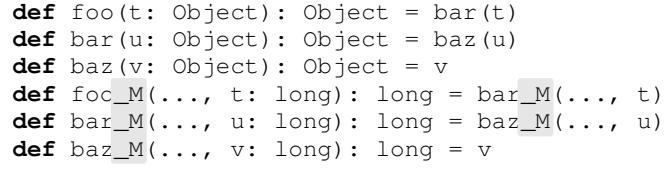

The translation shows that once execution entered the miniboxed path, by calling foo_M, it goes through without any boxing, only passing the value in the encoded (miniboxed) representation. Now let's see what happens if the @miniboxed annotation is removed:

def foo[@miniboxed $T$ ] ( $t: T): T=\operatorname{bar}(t)$

def $\operatorname{bar}[\mathrm{T}](\mathrm{U}: \mathrm{U}): \mathrm{U}=\operatorname{baz}(\mathrm{u})$

def baz[@miniboxed V] (v: V) : V = v

The bytecode produced is:

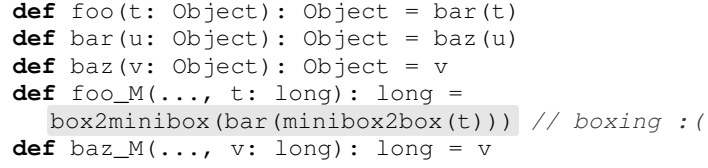

Two problems occur here:

- When method foo_M is called, it does not have a miniboxed version of bar to call further on, so it calls the erased one;

- When method bar is called, although baz has a miniboxed version, it cannot be called as the type information was erased.

These two problems correspond to the main two performance advisories: forward and backward warnings. A third one, related to data representation ambiguity, will be shown below.

Forward advisories. The first advisory (compiler warning) received by the programmer is also called a forward warning:

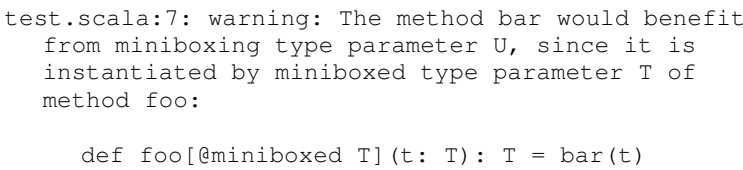

This advisory pushes the miniboxed representation from caller to callee when the arguments need to be boxed before being passed.

Backward advisories. The miniboxing annotation is also propagated from callee to caller:

test.scala:8: warning: The following code could benefit from miniboxing specialization if the type parameter $U$ of method bar would be marked as "@miniboxed U" (it would be used to instantiate miniboxed type parameter $\mathrm{V}$ of method baz) :

$\operatorname{def} \operatorname{bar}[\mathrm{U}](\mathrm{u}: \mathrm{U}): \mathrm{U}=\mathrm{baz}(\mathrm{u})$

Ambiguity advisories. Scala allows types to abstract over both primitives and objects. For example, wildcard types (known as existentials in Scala) can abstract over any type in the language. Any is the top of the Scala type system hierarchy, with two subclasses: AnyVal is the superclass of all value types (and thus primitives) while AnyRef is the superclass of all reference types, corresponding to Java's Object. Therefore, existentials, Any and AnyVal are not specific enough to pick a primitive or a reference representation. In this case, we issue a warning and box the values:

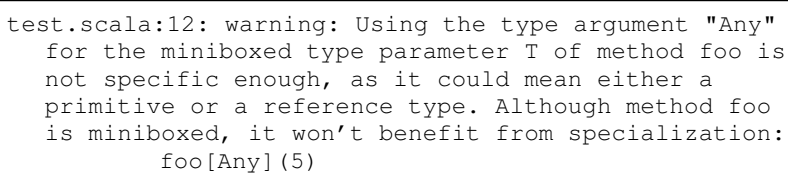

With these actionable warnings, even a novice programmer, not familiar to the miniboxing transformation, can still achieve the same performance as an expert manually sifting through the generated bytecode. We have several examples where programmers achieved speedups over $2 \mathrm{x}$ just by following the miniboxing advisories $[2,4,6]$. We will now explain the intuition behind generating performance advisories.

\subsection{Unification: Intuition}

The reason we chose to present the "forward", "backward" and "ambiguity" advisories is because, although they are only three of the ten cases, they are the warnings a typical programmer is most likely to encounter. They appear in all cases where a specialized variant of either a method or class needs to be chosen:

- Calling a miniboxed method;

- Instantiating a miniboxed class;

- Calling the method of a miniboxed class;

- Extending a miniboxed class or trait;

The one element common to all these cases is the need to pick the best matching miniboxed variant for the given type arguments. For example, given the method foo defined previously, for a call to foo [Int] (4), the compiler needs to find the best variant of foo and redirect the code to it. In this case, since the type argument of method foo is Int, which is a primitive type, and since the type parameter $\mathrm{T}$ in the definition of $\mathrm{foo}$ is marked as miniboxed, it will pick the foo_M variant, which uses the miniboxed representation. This operation is called unification, and we have unified the type parameter of foo, namely $\mathrm{T}$, and a type argument, Int. The unification algorithm is also responsible for issuing advisories.

Let us now focus on a more formal definition.

\subsection{Unification: Formalization}

Let us call the original method or class $\bigcirc$, with the type parameters $\mathrm{F} 1$ to $\mathrm{Fn}$ and $\mathrm{VO}$ the set of specialized variants corresponding to $\mathrm{o}$. Each specialized variant $\mathrm{v} \in \mathrm{V} O$ corresponds to a mapping from the type parameters to a representation in the set of \{miniboxed, reference, erased $\}$. Let us inverse this mapping, to produce another mapping from type parameters and representations to the specialized variants. Let's call it VS.

Then the unification algorithm can be reduced to choosing the corresponding $\mathrm{V} \in \mathrm{V} O$, for a term of type $\mathrm{O}[\mathrm{T} 1, \ldots, \mathrm{Tn}]$. This can be done following the algorithm in Figure 1.

Let us take an example to illustrate this:

class C[@miniboxed M, N] // $M$ is mboxed, $N$ is erased

class $D[L]$ extends $C[L$, Int $]$

When deciding which specialized variant of the miniboxed class $C$ to use as class D's parent, we have:

- the original class $\mathrm{O}=\mathrm{C}$;

- the type parameters $\mathrm{F} 1=\mathrm{M}$ and $\mathrm{F} 2=\mathrm{N}$; 
- the set of variants $\mathrm{VO}=\left\{\mathrm{C}_{-} \mathrm{M}, \mathrm{C}_{-} \mathrm{L}\right\}$;

- the inverse mapping VS $=\left\{\right.$ M: miniboxed and $\mathrm{N}$ : erased $\rightarrow \mathrm{C} \_\mathrm{M}$, M: reference and $\mathrm{N}$ : erased $\left.\rightarrow \mathrm{C}_{-} \mathrm{L}\right\}$

Now, applying the unification algorithm in Figure 1 for the type parameter $\mathrm{F} 1=\mathrm{M}$ coupled with the type argument $\mathrm{T} 1=\mathrm{L}$, it issues a forward warning followed by outputting (M: reference). Then, applying it to $\mathrm{F} 2=\mathrm{N}$ and $\mathrm{T} 2=\mathrm{Int}$, it issues a backward warning and outputs ( $\mathrm{N}$ : erased). From the two bindings, we obtain the specialized variant C_L to be a parent of D. Indeed, this is what happens in practice:

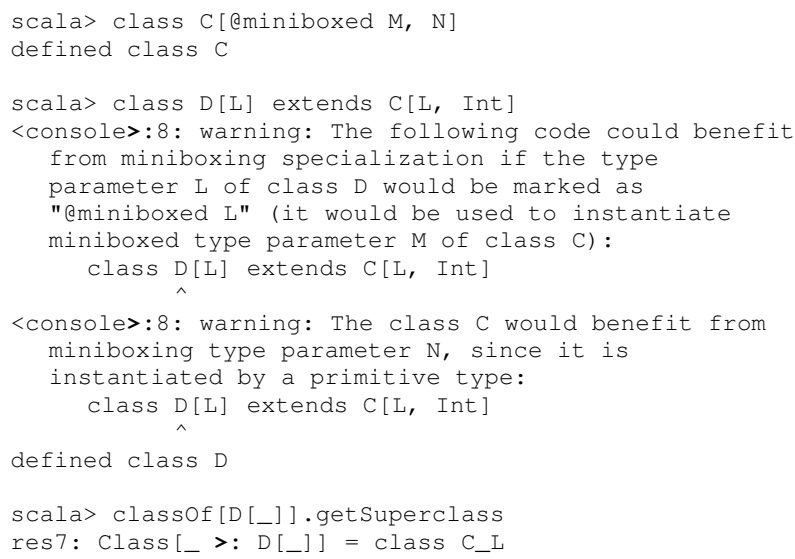

Now it is easy to guess where the forward and backward names come from: the direction in which the miniboxing transformation propagates between the type parameter and the type argument.

\subsection{Unification: Implementation}

The performance advisories are tightly coupled with the unification algorithm, which decides the variant that should be used for transforming the code. The processing is done one step at a time, with a type parameter and type argument pair. We will now show some issues that an implementer must be careful about.

Owner chain status. Since methods and classes in Scala can be nested in any order, we must be careful to propagate the status of the type parameters in the owner chain. In the following example:

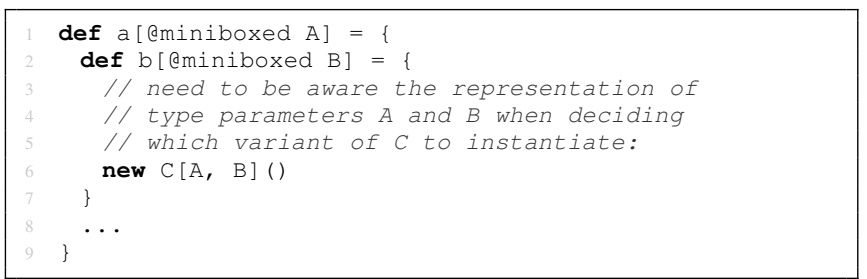

When deciding which miniboxed variant of class $\mathrm{C}$ to instantiate, we need to be aware of the nested methods we are located in as we duplicate and specialize the code: if we're in method b_M inside method a_M, we can rely on values of type $A$ and $B$ to be miniboxed. Contrarily, if we are in method $b$ inside method $a$, values of type $A$ and $\mathrm{B}$ are references.

Caching warnings. Instead of issuing warnings right away, they are being cached and later de-duplicated. The reason is that issuing too many warnings diminishes their value. Aside from the three advisories shown, there are special advisories dealing with the specialization transformation in Scala and certain library constructs that we show in the next section. Thus, we define an ordering of advisory priority and, if multiple warnings are cached, we only issue the most important ones.

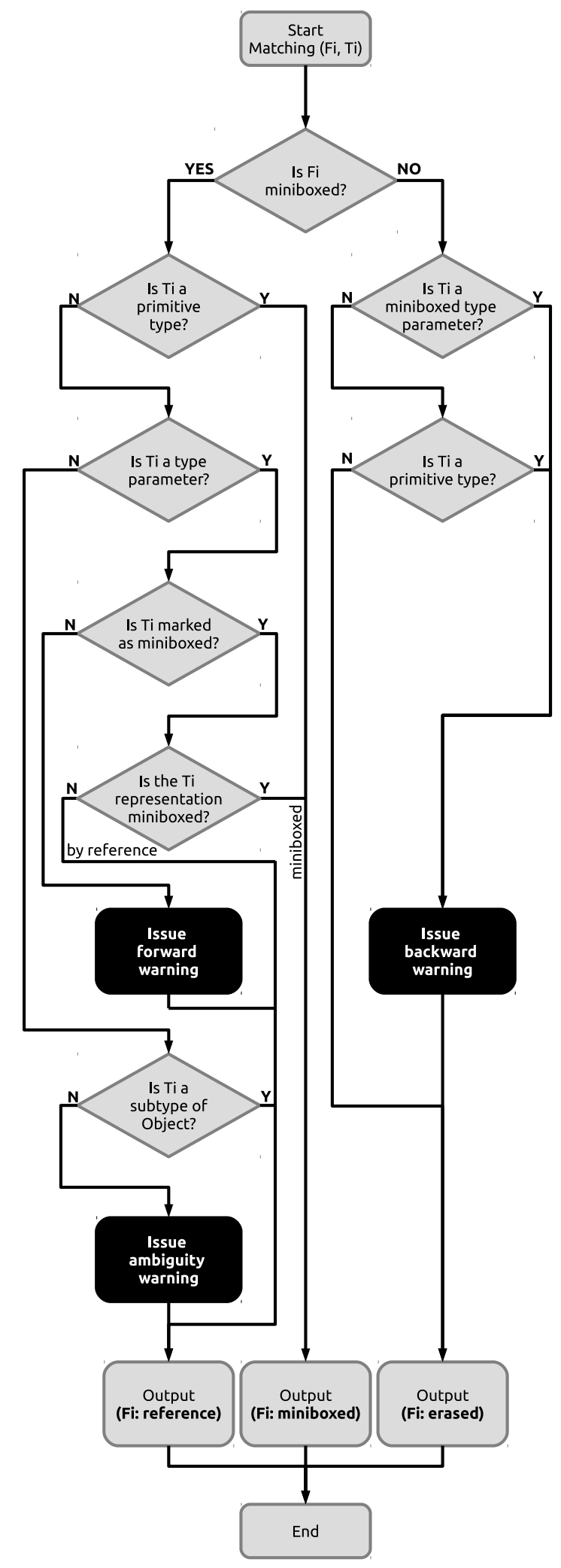

Figure 1: The unification algorithm for picking the data representation of a type parameter. 
Suppressing warnings. In certain scenarios, programmers are aware of their sub-optimal erased generic code but, due to compatibility requirements with other JVM programs or due to the fact that code lies outside the hot path, they chose not to change it. In these situations, they need to suppress the warnings, because instead of improving visibility, they might obscure other more important performance regressions in the program. However, a coarse-grained approach such as turning off all warnings is not desirable either, as it completely voids the benefit of advisories. For this scenario, the miniboxing transformation provides the egeneric annotation, which can suppress performance advisories:

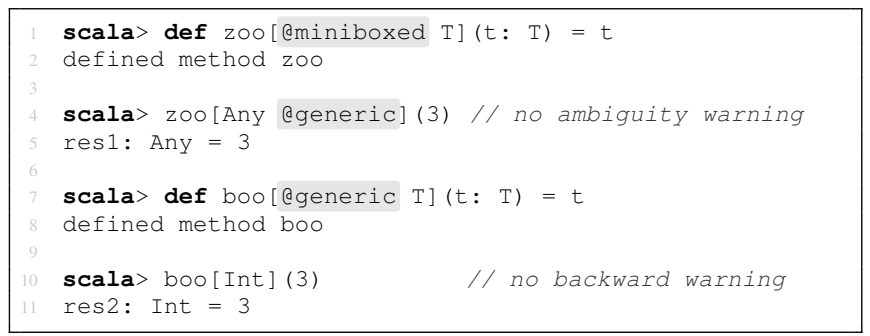

Libraries. In other cases boxing is caused by the interaction with erased generics from libraries. In this case, the default decision is not to warn, unless the programmer specifically sets the $-\mathrm{P}$ :minibox: warn-all compiler flag:

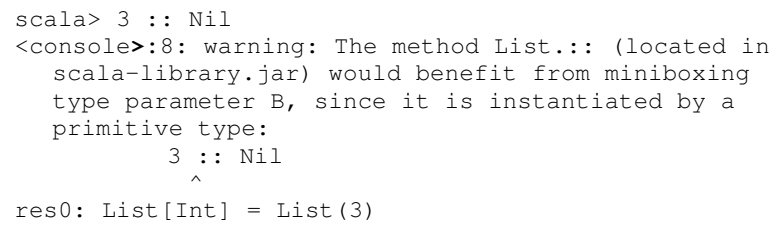

As we will see in the benchmarking section ( $\$ 5)$, the performance advisories allow programmers who are not familiar with the transformation to make the same changes an expert would do.

\section{Interoperating with Existing Libraries}

There is a clear parallel between the manual lambda specializations that are already in the Java Standard Library and thus cannot be eliminated and the specialized constructs in the Scala Standard Library, which cannot be replaced by a compiler plugin. Project Valhalla brings the ability to specialize generics to Java, while miniboxing brings a new compilation scheme for Scala generics. What is common between the two cases is the hard requirement that the new transformations work well with the existing constructs, which use different compilation schemes. This is the problem of interoperating with existing libraries.

In this section we will show how performance regressions occur when miniboxed code interacts with the Scala standard library, which uses either erased generics or the original specialization transformation. To counter these performance regressions, we show three approaches to efficiently bridge the gap between the miniboxing and specialization compilation schemes. Although this section mostly focuses on the interoperation between miniboxing and specialization, the techniques are general and can be applied to Java lambdas and Valhalla as well.

\subsection{The Interoperation Problem}

When interacting with the library from miniboxed code, the programmers forget the fact that library constructs, such as tuples and functions, do not share the same compilation scheme. Thus, they expect the same performance and flexibility as when using miniboxed classes. However, calling specialized code from miniboxed methods and vice-versa is not easy. For example:

def spec[@specialized $T$ ] ( $t$ : $T)$ : String = t.toString def mbox[@miniboxed $T$ ] (t: $T)$ : String $=\operatorname{spec}(t)$

The code is translated to:

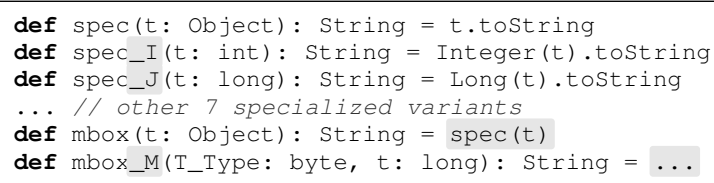

The reference-based mbox and spec methods can directly call each other, since there is a 1 to 1 correspondence. The problem is that, unlike these two methods, none of the specialized variants have a 1 to 1 correspondence to mbox_M. This only leaves the referencebased methods as candidates for the direct interoperation between miniboxing and specialization.

Although it may seem like mbox_M could directly invoke spec_J, since the argument types match, this would be incorrect, as the value $t$ in mbox_M can be any primitive type, encoded as a long, whereas $t$ in spec_J can only be a long integer. Thus, if we were to call spec_J from mbox_M passing an encoded boolean, instead of returning either "true" or "false", it would return the encoded value of the boolean.

The mbox_M method has one more piece of information: T_Type, the type byte describing the encoded primitive. In theory, the miniboxed method could use this type byte to dispatch the right specialized counterpart:

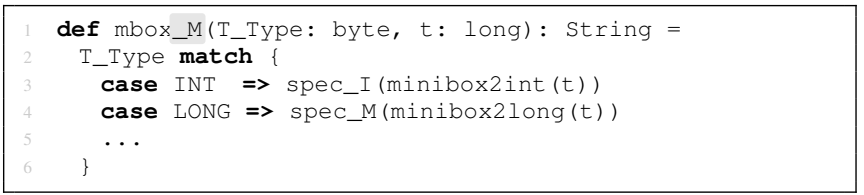

Although this indirect approach seems to work and can easily be automated, it is actually a step in the wrong direction: the miniboxing transformation would be introducing extra overhead without offering the programmer any feedback on how and why this happens. Furthermore, when multiple type parameters are specialized, all $10^{N}$ possible combinations would have to be added to the match, making it very large. This is likely to confuse the Java Virtual Machine inlining heuristics, causing severe performance regressions.

It may seem like the other way around would be easier: allowing specialized code to call miniboxed methods without performing a switch. However this is not the case because, having been developed first, specialization is not aware of miniboxing. Thus, when calling miniboxed methods, specialization invokes the reference version, boxing the arguments and unboxing the returned value.

With this in mind, our decision was to go with simplicity and symmetry: the bridge between miniboxing and specialization goes through boxing. To allow transparency, miniboxing issues performance advisories about specialized code that should be miniboxed:

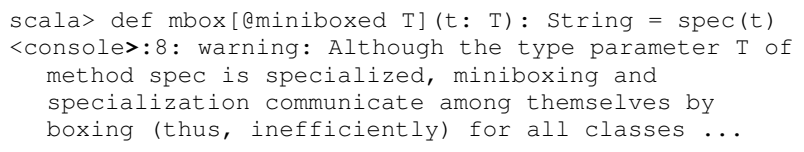




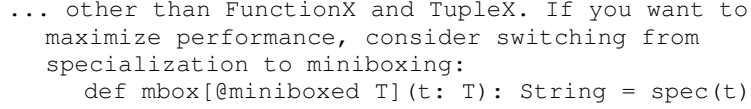

This solution works well with most of the code that lies within the programmer's control, including for the case where 3rd party libraries distribute both a specialized and a miniboxed version. However, the one library which cannot have multiple versions and happens to use specialization is the Scala standard library. The two most wide-spread constructs affected by this are Tuples and Functions, both of which are specialized. This makes the following function a worst-case scenario for vanilla miniboxing:

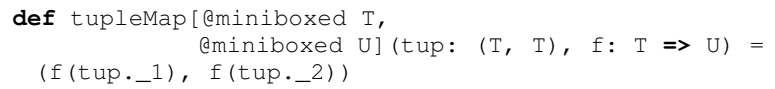

Despite the annotations, with the vanilla miniboxing transformation, all versions of the tupleMap method use reference-based tuple accessors and function applications, leading to slow paths irreversibly creeping into miniboxed code. For many applications, this is a no-go, so our task was to eliminate these slowdowns. In the following subsection we present three possible approaches and show where each works best.

\subsection{Eliminating the Interoperation Overhead}

We show three approaches to eliminating the boxing overhead when calling specialized code from miniboxed classes or methods.

Accessors. The simplest answer to the problem of inter-operating with specialization is to switch on the type byte, as shown previously. To avoid confusing the Java Virtual Machine inlining heuristics, we can extract the operation into a static method, that we call separately. This approach needs to be implemented both for accessors, allowing the specialized values to be extracted directly into the miniboxed encoding and for constructors, allowing miniboxed code to instantiate specialized classes without boxing. This is the approach taken for Tuple classes ( $\$ 4.3)$;

Transforming objects. The accessors approach allows us to pay a small overhead with each access. This is a good trade-off when the constructs are only accessed a couple of times during their lifetime, which is the case for tuples. In other cases, such as functions, the apply method is presumably called many times during the object lifetime, making it worthwhile to completely eliminate the overhead. In this case, a better approach is to replace the Function objects by MiniboxedFunctions, introducing conversions between them where necessary. This way, the apply method exposed by MiniboxedFunction can be called directly, and this can compensate for a potentially greater cost of constructing the MiniboxedFunction object. This way, switching on the type bytes is done only once, when converting the function, and then amortizes over the function lifetime ( $\$ 4.4)$;

New API. In some cases, the API and guarantees are hardcoded into the platform. This is the case for the Scala Array class, for which the original miniboxing plugin chose the accessors approach [31]. However, a better tradeoff is achieved by defining a new MbArray class with a similar API but different guarantees. This approach will be briefly mentioned in the Arrays subsection ( 4.5$)$.

The next sections discuss the three methods above.

\subsection{Tuple Accessors}

The Scala programming language offers a very concise and natural syntax for library tuples, allowing users to write $(3,5)$ in- stead of the desugared new Tuple2[Int, Int] $(3,5)$. Similarly, it allows programmers to write (Int, Int) instead of Tuple2[Int, Int]. If we were to introduce miniboxed tuples, we would not be able to use the syntactic sugar to express programs, losing the support of many programmers. Instead, a better choice is to efficiently access specialized Scala tuples.

Although we don't have statistically significant data, our experience suggests that Tuple classes have their components accessed only a few times during their life. Therefore, both for compatibility reasons and to avoid costly conversions, we decided to allow the Tuple class to remain unchanged, instead focusing on providing accessors and constructors that use the miniboxed encoding.

The optimized tuple accessors are written by hand and are explicitly given the type byte:

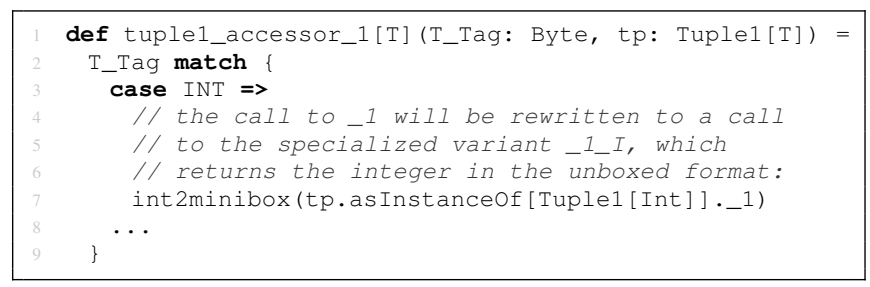

Once the tuple is cast to a Tuple1 [ Int ], the specialization transformation kicks in and transforms the call to _ 1 into a specialized call to _1_I, the integer variant. Since the int 2 minibox conversion also takes an unboxed integer, the overhead of boxing is completely eliminated.

The specialized constructors are motivated by two observations: (1) allocating tuples in the miniboxed code without special support requires boxing and, even worse (2) the tuples created use the reference-based variant of the specialized class, thus voiding the benefits of having added tuple accessors. The code for the tuple constructors is also written by hand and is very similar to the accessor code: it dispatches on the type tags to create tuples of primitive types, which specialization can rewrite to the optimized variants.

Introducing accessors and constructors is done by the miniboxing plugin when encountering a tuple access followed by a conversion to the miniboxed representation or when the tuple constructor is invoked with all the arguments being transformed from the miniboxed representation to the boxed one. There are two reasons this step needs to be automated:

- By default, programmers do not have access to the type bytes directly, as this would allow them to introduce unsoundness in the type system (they can inspect their representation using miniboxing reflection, but this is outside the scope);

- One of the reasons tuples are useful is their great integration with the language, allowing a very concise syntax. Asking programmers to use anything other than this syntax would be as bad as developing our own, no-syntax-sugar miniboxed tuple.

With these three changes, benchmarks show a $2 \mathrm{x}$ speedup when accessing tuples and a 5\% slowdown compared to the equivalent code which accesses the tuples directly. The benchmark we used was a tuple quicksort algorithm $(\S 5)$.

With the three elements above, accessors, constructors and the automatic code rewriting we create a direct bridge between specialized tuples and miniboxed classes. Unfortunately, as we've seen before, adding such accessors has to be a carefully-weighted, contextspecific decision, so automating it would not provide much benefit. For example, this choice would not be suitable for functions. 


\subsection{Functions}

Like tuples, functions in Scala have a concise and natural syntax, which ultimately desugars to one of the FunctionX traits, where $\mathrm{X}$ is the function arity. For example:

val $\mathrm{f}:$ Int $\Rightarrow$ Int $=(\mathrm{x}:$ Int $) \Rightarrow \mathrm{x}+1$

Desugars to:

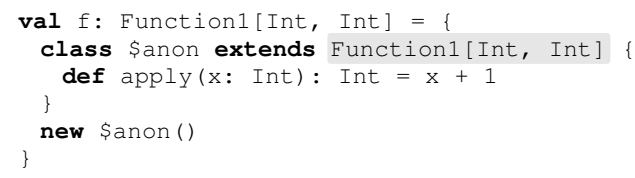

Since Function objects are specialized, the code is compiled to:

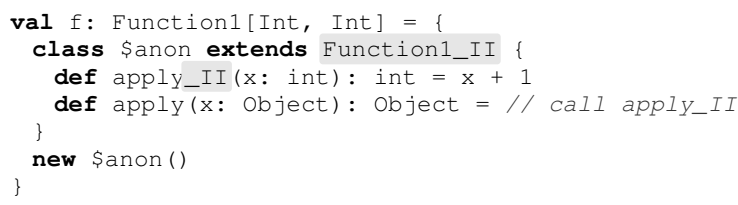

When interoperating with miniboxed code, functions can only use the reference-based apply, introducing performance regressions.

In our early experiments on transforming the Scala collections hierarchy using the miniboxing transformation [17], we were proposing an alternative miniboxed function trait, called MbFunction, and were performing desugaring by hand. The performance obtained was good, but desugaring by hand was too tedious. Later on, we received a suggestion from Alexandru Nedelcu stating that, since functions in Scala are specialized, we should be able to interface directly, thus benefiting from the desugaring build into Scala without paying for the boxing overhead.

Our initial approach used accessors, but we soon learned that switching on as many as 3 type bytes with each function application incurs a significant overhead. Instead, we decided to re-introduce MbFunctionX within the code compiled by the miniboxing plugin, where $\mathrm{X}$ is the arity and can range between 0 and 2 (Scala includes functions with arities up to 22 , but arities above 2 are no longer specialized). Yet, this time the MbFunctionX objects would be introduced automatically.

Code transformation. The miniboxing plugin automatically transforms Functionx to MbFunctionX:

- All references to FunctionX are converted to MbFunctionX;

- Function definitions create MbFunct ionX instead of Funct ionX;

For example, the code:

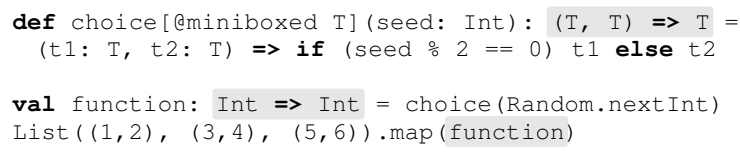

Is transformed into:

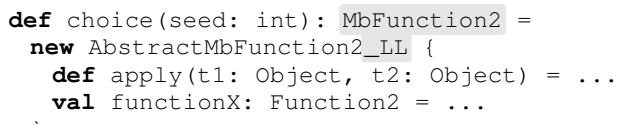

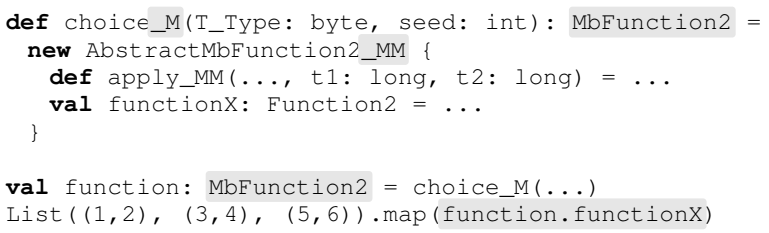

Explaining how the code transformation works is beyond the scope of this paper and has been thoroughly studied in previous literature $[32,33]$. The result is that, within miniboxed code, only the $\mathrm{MbF}$ unctionX representation is used. FunctionX is only referenced in a limited number of cases:

- When miniboxed code needs to pass a function to pre-miniboxing code (which uses the FunctionX representation);

- When miniboxed code receives a function from pre-miniboxing code (using the FunctionX representation);

- When a miniboxed class or method extends a pre-miniboxed entity that takes FunctionX arguments;

- When an MbFunctionX value is assigned to supertypes of FunctionX, it needs to be converted;

Conversions can occur in both directions, from Functionx objects to MbFunctionX and back.

Converting FunctionX objects to their miniboxed counterparts is done using switches that allow the newly created MbFunctionX to directly call the unboxed apply, fără boxing:

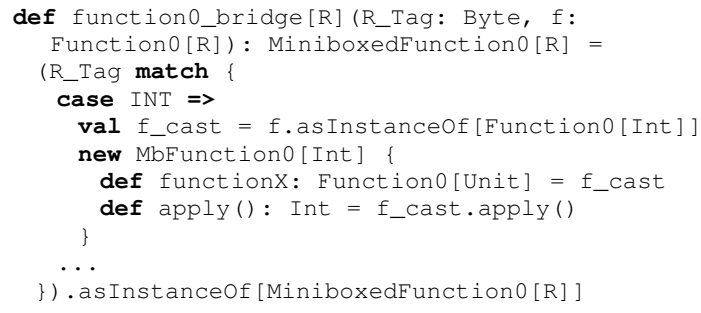

In the above code, $f$ is statically known to be of type Function [ Int ], thanks to the type byte. This allows the code to introduce f_cast, which in turn allows the specialization transformation to rewrite the call from the reference-based apply to the unboxed apply_I. On its side, miniboxing instantiates MbFunctiono_M instead of MbFunction 0 and moves the code to the specialized apply_M method. With these rewrites, the anonymous MbFunction instance can call the underlying function without boxing:

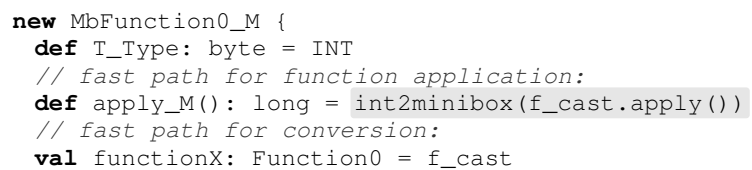

Converting MbFunctionX objects to FunctionX easy, since each MbFunctionX object contains its FunctionX counterpart in the functionx field.

By transforming the function representation, we have eliminated the overhead of calling functions completely. Furthermore, using the previous two strategies to minimize the conversion overhead, we enabled function-heavy applications to achieve speedups between 2 and $14 x[1,33]$. 


\begin{tabular}{|l|c|c|}
\hline Benchmark & Generic & Miniboxed \\
\hline Builder & $161.61 \mathrm{~s}$ & $53.56 \mathrm{~s}$ \\
Map & $98.43 \mathrm{~s}$ & $49.38 \mathrm{~s}$ \\
Fold & $87.98 \mathrm{~s}$ & $46.14 \mathrm{~s}$ \\
Reverse & $27.97 \mathrm{~s}$ & $33.84 \mathrm{~s}$ \\
\hline
\end{tabular}

Table 1: RRB-Vector operations for $5 \mathrm{M}$ elements.

\subsection{Arrays}

The array transformation [9] is beyond the scope of this paper, but we included it as a good example for using performance advisories.

The Array bulk storage in Scala makes certain assumptions that are not compatible with miniboxing, leading to performance regressions in some corner cases. To address this limitation, we introduced a new type of array, dubbed MbArray, which integrates very well within the miniboxing transformation. However, since the MbArray guarantees do not match the ones offered by Scala arrays, we cannot automate the transformation. Instead, we issue performance advisories to inform programmers about MbArray:

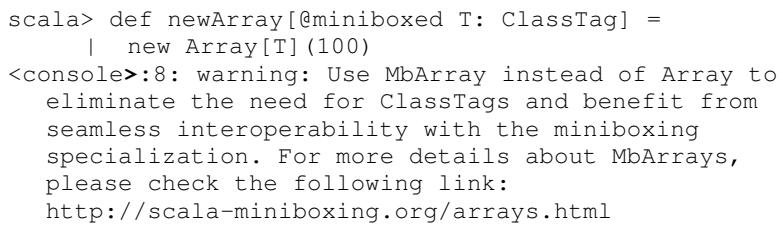

This concludes the three approaches to interoperating with the specialized Scala library.

\section{Benchmarks}

In this section we show three different scenarios where miniboxing has significantly improved performance of user programs. We specifically avoid mentioning benchmarking methodology, as each of the experiments was ran on a different setup. Yet, all three examples show a clear trend: the techniques explained in the paper improve both performance and the programmer experience.

The RRB-Vector data structure [22, 29] is an improvement over the immutable Vector, allowing it to perform well for data parallel operations. Currently, the immutable vector collection in the Scala library offers very good asymptotic performance over a wide range of sequential operations, but fails to scale well for data parallel operations. The problem is the overhead of merging the partial results obtained in parallel, due to the rigid Radix-Balanced Tree, the Vector's underlying structure. Contrarily, the RRB-Vector structure uses Relaxed Radix-Balanced (RRB) Trees, which allow merges to occur in effectively constant time while preserving the sequential operation performance. This enables the RRB-Vect or to scale linearly with the number of cores when executing data parallel operations. Thanks to its improved performance, the RRB-Vector data structure is slated to replace the vector implementation in the Scala library in a future release.

\begin{tabular}{|l|c|c|c|}
\hline Benchmark & Generic & $\begin{array}{c}\text { Miniboxed } \\
\text { some } \\
\text { advisories } \\
\text { heeded }\end{array}$ & $\begin{array}{c}\text { Miniboxed } \\
\text { all } \\
\text { advisories } \\
\text { heeded }\end{array}$ \\
\hline 1st run & $4192 \mathrm{~ms}$ & $3082 \mathrm{~ms}$ & $1346 \mathrm{~ms}$ \\
2nd run & $4957 \mathrm{~ms}$ & $2998 \mathrm{~ms}$ & $1187 \mathrm{~ms}$ \\
3rd run & $4755 \mathrm{~ms}$ & $3017 \mathrm{~ms}$ & $1178 \mathrm{~ms}$ \\
4th run & $3969 \mathrm{~ms}$ & $2535 \mathrm{~ms}$ & $1094 \mathrm{~ms}$ \\
5th run & $4073 \mathrm{~ms}$ & $2615 \mathrm{~ms}$ & $1163 \mathrm{~ms}$ \\
\hline
\end{tabular}

Table 2: Speedups based on performance advisories, PNWScala

\begin{tabular}{|l|c|}
\hline Transformation & Running time \\
\hline Generic & $684.4 \mathrm{~ms}$ \\
Miniboxed (no tuple support) & $726.8 \mathrm{~ms}$ \\
Miniboxed (with tuple support) & $323.2 \mathrm{~ms}$ \\
Specialized & $322.5 \mathrm{~ms}$ \\
Monomorphic & $318.1 \mathrm{~ms}$ \\
\hline
\end{tabular}

Table 3: Sorting 1M tuples using quicksort.

The original RRB-Vector implementation used erased generics. To show that performance advisories can indeed guide developers into improving performance, we asked a Scala developer who was not familiar with the RRB-Vector code base to switch the compilation scheme to miniboxing. Before handing in the code, we removed the parallel execution support [5], reducing the code base by $30 \%$. Then, the developer compiled the code with the miniboxing plugin, which produced 28 distinct warnings. These warnings guided the addition of aminiboxed annotations where necessary and the introduction of MbArray objects instead of Scala arrays. By following the performance advisories, in less than 30 minutes of work, our developer managed to improve the performance of the $\mathrm{RRB}-\mathrm{Vect}$ or operations by up to $3 \mathrm{x}$. A counter-intuitive effect was that it took three rounds of compiling and addressing the warnings before the improvement was visible: each iteration introduced more @miniboxed annotations, in turn triggering new warnings, as new methods could benefit from the annotation.

Table 1 shows the performance improvements measured on four key operations of the RRB-Vector: creating the structure element by element using a builder and invoking bulk data operations: map, fold and reverse. The ScalaMeter framework [24] was used as a benchmarking harness on a quad-core Intel Core i7-4600U processor running at $2.10 \mathrm{GHz}$ with $12 \mathrm{~GB}$ of RAM, on OpenJDK7.

The numbers show speedups between 1.9 and $3 \mathrm{x}$ for the builder, map and fold benchmarks. This can be explained by the fact that, in the erased version, each element required at least a boxing operation, and thus a heap allocation. On the other hand, the reverse operation does not require any boxing so there is no speedup achieved. Nevertheless, introducing the miniboxing transformation does not lead to significant slowdowns.

If we consider the RRB-Vector development time, which took four months of work and resulted in $\sim 3 \mathrm{~K}$ lines of source code, the performance advisories issued by the miniboxing plugin allowed a new developer, with no knowledge of the code base, to deploy the miniboxing transformation in a negligible period of 30 minutes, producing speedups of up to $3 \mathrm{x}$.

Image processing. Performance advisories can be used to improve the performance of Scala programs without any previous knowledge of how the transformation works. This was shown at the PNWScala 2014 developer conference [3], where Vlad Ureche presented how the miniboxing plugin guides the programmer into improving the performance of a mock-up image processing library by as much as $4 \mathrm{x}$ [4]. The presentation was recorded and the performance numbers are included in Table 2 for quick reference.

Tuple accessors have been tested by implementing a tuple sorting benchmark using a generic quicksort algorithm. Table 3 shows the results for sorting 1 million tuples based on their first element. We used different transformations for the generic quicksort algorithm: first, we benchmarked the erased generics performance, which, as expected, were slow. Surprisingly, the miniboxed version without tuple support was even worse, $7 \%$ slower than erased generics. Then, adding tuple accessor support to the miniboxing transformation improved the performance by $2 \mathrm{x}$, making it comparable to the original specialization transformation and to the monomorphic (non-generic, hand specialized) version of the quicksort algorithm. 


\section{Related Work}

The most significant related work lies in the area of run-time profilers which can offer feedback at the language level. We would like to point the work of St-Amour on optimization feedback [27] and feature-based profiling [28]. Profiling has existed for a long time at lower levels, such as at the Java Virtual Machine level, with profilers such as YourKit [8] or the Java VisualVM [7] or the x86 assembly, with processor hardware counters.

The area of opportunistic optimizations has seen an enormous growth thanks to dynamic languages such as JavaScript, Python and Ruby, which require shape analysis and optimistic assumptions on the object format to maximize execution speed. We would like to highlight the work of Mozilla on their *Monkey JavaScript VMs [16], Google's V8 JavaScript VM and the PyPy Python virtual machine $[10,11]$. While this is just a short list of highlights, the Truffle compiler [34, 35] is now a general approach to writing interpreters that make optimistic assumptions, allowing maximum performance to be achieved by partially evaluating the interpreter for the program at hand, essentially obtaining a compiled program thanks to the first Futamura projection [15].

In the area of data representation, this work assumes familiarity with specialization [13] and miniboxing [2, 31]. The program transformation which enables the functions to be transformed into miniboxed functions is thoroughly discussed in $[32,33]$. There has been previous work on miniboxing Scala collections [17] and on unifying specialization and reified types [30]. We have also seen a revived interest in specialization in the Java community, thanks to project Valhalla, which aims at providing specialization and value class support at the virtual machine level $[18,25]$. In the Java 8 Micro Edition functions are also represented differently [23].

\section{Conclusion}

This paper shows several approaches to allowing different generics compilations schemes to interoperate without incurring performance regressions:

- Harmonizing the generics compilation scheme thanks to actionable performance advisories;

- Bridging the gap between library constructs that use different generics compilation schemes, specifically:

- The accessor approach;

- The replacement approach;

- The advisory-based approach.

The implementation results are validated using the miniboxing plugin, which automates the approaches described, showing performance improvements between $2 \mathrm{x}$ and $4 \mathrm{x}$.

\section{Acknowledgements}

The authors are grateful to the following people who motivated the development of the features described in the paper: Alexandru Nedelcu, Aymeric Genet and Aggelos Biboudis (Functions), Philip Stutz, Stu Hood, Iulian Dragos and Rex Kerr (warnings), Julien Truffaut (tuple accessors). We are thankful to Vincent St-Amour for the ground-breaking work on program optimization advisories.

We would like to thank Brian Goetz, Maurizio Cimadamore and the PPPJ reviewers for their helpful comments and suggestions.

\section{References}

[1] ildl Compiler Plugin Documentation. URL https://github.com/ miniboxing/ildl-plugin/wiki.

[2] The Miniboxing plugin website. URL http://scala-miniboxing.org.

[3] PNWScala Conference, . URL http://pnwscala.org.

[4] PureImage Library Optimization,. URL http://scala-miniboxing. org/example_pureimage.html.
[5] RRB-Vector benchmarks. URL https://github.com/ milosstojanovic/miniboxing-plugin/tree/rrbvector/ tests/lib-bench/src/miniboxing/benchmarks/rrbvector.

[6] Optimistic Respecialization Attempt 6. URL http://io.pellucid.com/ blog/optimistic-respecialization-attempt-6.

[7] Java VisualVM. URL https://visualvm. java.net/.

[8] YourKit Profiler. URL https://www.yourkit.com/java/profiler/.

[9] R. M. Beguet. Miniboxing and the MbArray API. Technical report, EPFL, 2015. https://infoscience.epfl.ch/record/208957.

[10] C. F. Bolz, A. Cuni, M. Fijalkowski, and A. Rigo. Tracing the Meta-level: PyPy's Tracing JIT Compiler. In ICOOLPS. ACM, 2009.

[11] C. F. Bolz, L. Diekmann, and L. Tratt. Storage Strategies for Collections in Dynamically Typed Languages. In OOPSLA. ACM, 2013.

[12] G. Bracha, M. Odersky, D. Stoutamire, and P. Wadler. Making the future safe for the past: Adding Genericity to the Java Programming Language. In OOPSLA. ACM, 1998.

[13] I. Dragos. Compiling Scala for Performance. PhD thesis, École Polytechnique Fédérale de Lausanne, 2010.

[14] I. Dragos and M. Odersky. Compiling Generics Through User-Directed Type Specialization. In ICOOOLPS, Genova, Italy, 2009.

[15] Y. Futamura. Partial Evaluation of Computation Process-An Approach to a Compiler-Compiler. Higher-Order and Symbolic Computation, 1999.

[16] A. Gal. Trace-based Just-in-time Type Specialization for Dynamic Languages. In PLDI. ACM, 2009.

[17] A. Genêt, V. Ureche, and M. Odersky. Improving the Performance of Scala Collections with Miniboxing (EPFL-REPORT-200245). Technical report, EPFL, 2014. URL http://scala-miniboxing.org/.

[18] B. Goetz. State of the Specialization, 2014. URL http://web.archive. org/web/20140718191952/http://cr.openjdk. java.net/ rbriangoetz/valhalla/specialization.html.

[19] B. Goetz. Announcement, July 2015. URL https://web.archive. org/web/20150808172443/http://mail.openjdk. java.net/ pipermail/valhalla-dev/2015-July/001245.html.

[20] B. Goetz. Description, August 2015. URL https://web.archive. org/web/20150808172447/http://mail.openjdk. java.net/ pipermail/valhalla-dev/2015-August/001295.html.

[21] A. Kennedy and D. Syme. Design and Implementation of Generics for the .NET Common Language Runtime. In PLDI, 2001.

[22] V. U. N. Stucki, P. Bagwell and T. Rompf. RRB Vector: A Practical General Purpose Immutable Sequence. ICFP, 2015.

[23] O. Pliss. Closures on Embedded JVM. JVM Languages Summit, Santa Clara, CA, august 2014.

[24] A. Prokopec. ScalaMeter. URL http://axel22.github.com/ scalameter/.

[25] J. Rose. Value Types and Struct Tearing,. URL https://web. archive.org/web/20140320141639/https://blogs.oracle. com/jrose/entry/value_types_and_struct_tearing.

[26] J. Rose. Value Types in the VM, . URL http: / /web. archive.org/web/ 20131229122932/https://blogs.oracle.com/jrose/entry/ value_types_in_the_vm.

[27] V. St-Amour, S. Tobin-Hochstadt, and M. Felleisen. Optimization Coaching: Optimizers Learn to Communicate with Programmers. In OOPSLA'12, 2012.

[28] V. St-Amour, L. Andersen, and M. Felleisen. Feature-Specific Profiling. In CC'15, 2015.

[29] N. Stucki. Turning Relaxed Radix Balanced Vector from Theory into Practice for Scala Collections (Master Thesis). Master's thesis, EPFL, 2015.

[30] N. Stucki and V. Ureche. Bridging islands of specialized code using macros and reified types. In SCALA. ACM, 2013.

[31] V. Ureche, C. Talau, and M. Odersky. Miniboxing: Improving the Speed to Code Size Tradeoff in Parametric Polymorphism Translations. In OOPSLA, 2013.

[32] V. Ureche, E. Burmako, and M. Odersky. Late Data Layout: Unifying Data Representation Transformations. In OOPSLA '14. ACM, 2014.

[33] V. Ureche, A. Biboudis, Y. Smaragdakis, and M. Odersky. Automating Ad hoc Data Representation Transformations. In OOPSLA. ACM, 2015.

[34] T. Würthinger, A. Wöß, L. Stadler, G. Duboscq, D. Simon, and C. Wimmer. SelfOptimizing AST interpreters. In DLS. ACM, 2012.

[35] T. Würthinger, C. Wimmer, A. Wöß, L. Stadler, G. Duboscq, C. Humer, G. Richards, D. Simon, and M. Wolczko. One VM to Rule Them All. In Onward! ACM, 2013.

[36] D. Yu, A. Kennedy, and D. Syme. Formalization of Generics for the .NET Common Language Runtime. In POPL, POPL '04. ACM, 2004. 